\title{
Elevated Serum Level of DHEAS as a Hormone and IL-6 as a Proinflammatory Cytokine May Better Indicate Metabolic Syndrome in PCOS Women
}

\author{
Zozan Guleken $^{1^{*}} \quad$ Huri Bulut $^{2} \quad$ Pınar Yalçın Bahat ${ }^{3} \quad$ Sibel Yılmaz $^{3} \quad$ Devrim Sarıbal $^{4^{*}}$ \\ 1.Department of Physiology, Uskudar University Faculty of Medicine, Istanbul, Turkey \\ 2.Department of Medical Biochemistry, Faculty of Medicine Istinye University, Istanbul, Turkey \\ 3.Health Science University Istanbul Kanuni Sultan Suleyman Training and Research Hospital, Department of \\ Obstetrics and Gynecology \\ 4.Department of Biophysics, İstanbul University-Cerrahpaşa, Cerrahpaşa Medical School, Istanbul, Turkey
}

\begin{abstract}
Polycystic ovarian syndrome (PCOS) is the most prevalent metabolic disorder in reproductive-age women, indeed multifactorial condition. Cytokines such as TNF-alpha and IL-6 have influence on several metabolic activities. The increase in dehydroepiandrosterone (DHEA) is thought to play an important role in IL-6-mediated pathogenetic effects in PCOS women. The aim of this study is to explore whether TNF-alpha and IL-6 are correlated hormone levels in PCOS disease. We recruited 65 PCOS patients and 45 healthy controls. The serum IL-6, TNF-alpha, levels were measured by ELISA and clinical characteristics were measured. Both serum IL-6, TNF-alpha levels $(\mathrm{p}<0.01)$, or the serum TSH, FSH, LH, PRL levels were significantly higher in the patients of PCOS $(\mathrm{p}<0.01)$. Serum IL-6 levels were correlated with DHEAS level $(\mathrm{r}=-0.4186, \mathrm{p}<0.01)$. DHEAS level tended to be higher in PCOS women with a correlation between age, BMI, IL-6 level and positively correlated with FSH, LH and PRL levels ( $<<0.01)$. There was an elevation of TSH level in the PCOS group $(2.4 \pm 1.5)$ vs. control group (1.8 \pm 1.6$)$. However, in control samples, there was no correlation between TNF alpha and IL-6, while in the PCOS samples positive correlation, was obtained. Oral glucose tolerance test (OGTT) was higher than the control (20.38 \pm 13.2$)$ group vs. PCOS women $(128.33 \pm 33.8)$ but only $3.44 \%$ of them had Type II diabetes. HOMA-IR index was higher in the PCOS group $(8.26 \pm 15.06)$ vs. control group $(2.12 \pm 0.88)$ too. There was a correlation between age and IL-6 level and BMI. Serum IL-6 and DHEAS levels were significantly altered in PCOS patients and determined which might be PCOS prognostic importance.
\end{abstract}

Keywords; PCOS; Cytokines; TNF-alpha; IL-6; DHEAS; Metabolic syndrome

DOI: $10.7176 / \mathrm{JMPB} / 71-02$

Publication date:June $30^{\text {th }} 2021$

\section{Introduction}

Polycystic ovary syndrome (PCOS) is an endocrine abnormality in women of reproductive age with the prevalence ranging from 9 to $18 \%$ (1). The disease is mainly is determined by the disfunction of ovulation, resistance of insulin, excess of androgens in circulation (2). The pathophysiology of the disease is still unknown but there is high evidence that chronic inflammation and oxidative stress may have a significant role in the pathophysiology of PCOS $(3,4)$. Cytokines soluble proteins which are small molecules are produced in response to antigens. They are initially defined as mediators for regulating the adaptive immune systems (5). Cytokines serve as chemical messengers such as tumor necrosis factors (TNF), interleukins (IL), interferons, chemokines, and growth factors (ㅁ). They mediate in intracellular signaling pathways which are characterized by proinflammatory and anti-inflammatory effects (7). TNF- $\alpha$ is a well-considered pro-inflammatory cytokine and IL-6, is considered to have both pro-and anti-inflammatory actions (). Beyond IL-6 is known for its immunological importance, IL-6 pointed as a molecule that acts at various physiological and pathological levels in different systems and processes as well energy balances and metabolism (9). It is known that TNF- $\alpha$, IL-6 has an important role in an inflammatory response which may induce phospholipases, sphingomyelinases, and concomitantly proteolyze phosphatidylcholine and sphingomyelin synthesizing enzymes as well as effects on the phospholipid metabolism (10). Cytokine production from various tissues generally induced by free oxygen radicals which are mediators of signal transduction pathways (11). It is reported that they have a potential role to inhibit the activation of oxidant-mediated transcription factors as well as preventing the transcriptional activation of inflammatory cytokines (12). Imbalance in metabolic status is commonly associated with changes in metabolic rate related to oxygen consumption which may lead to enhancement of the generation of oxidative stress status (13). Chronic inflammation is characterized by an increase in proinflammatory cytokines and chemokines which is associated with insulin resistance (14). There are researches have revealed that serum oxidative markers are significantly increased in patients with PCOS compared with the normal (15). Therefore, supplementation with compounds containing anti-inflammatory and antioxidant properties may be effective in improving the pathological condition of PCOS (16). In the light of the foregoing consideration, we aimed to 
compare circulating hormone levels by cytokines to contribute the relation between hormone concentrations and inflammation in PCOS women.

\section{Methods}

\subsection{Subjects}

A total of 110 subjects recruited 65 diagnosed with PCOS and 45 healthy females served as control subjects aimed to match BMI and age among groups. PCOS diagnostic evaluation and biological materials of the PCOS diagnosed patients were performed and provided by two expert physicians working in the field. Healthy control groups were selected according to inclusion criteria who had not to have both a history of infertility, hysterectomy or ovariectomy, or menstrual problems and hyperandrogenism signs. The participants were recruited from the attending Department of Obstetrics and Gynecology in Health Science University at Kanuni Sultan Suleyman Training and Research Hospital in İstanbul with the approval of the Ethics Committee and permission (date 2021.03 and number 85) depending on the guide of Helsinki Declaration. The data collected from patients who were involved in this experiment were subject to the Personal Data Act and Act on Ethical Review for research involving human subjects. All research participants gave their permission to be part of a study. BMI was calculated as $\mathrm{kg} / \mathrm{m}^{2}$. The details of participants are depicted in Table1.

\subsection{Laboratory methods}

\subsubsection{TNF- $\alpha$ ELISA}

TNF- $\alpha$ ELISA kit (Elabscience C., Cat no: E-EL-R0019, China) was used for the quantitative measurements Thermo Scientific Microplate Reader (USA) was used for the measurements at $450 \mathrm{~nm}$ with the kit detection was $78.13-5000 \mathrm{pg} / \mathrm{mL}$.

\subsubsection{IL-6 ELISA}

Quantitative measurements of IL-6 in serum samples were carried out by using IL-6 ELISA kit (Elabscience, EEL-H0102, USA). Optical density was measured at $450 \mathrm{~nm}$ via Thermo Scientific Microplate Reader (USA) and with kit detection range was 7.81-500 pg/mL.

\section{Statistical analysis}

Data were analyzed using Statistical Package for Social Science version 17 (SPSS Inc., Chicago, IL) and were expressed as mean $\pm \mathrm{SD}$. The concentration of parameters was compared between the groups using repeated measures of The Analysis of Variance (ANOVA) test followed with Bonferroni. Correlation analyses were performed using Spearman's correlation coefficient. The difference was considered statistically significant at $\mathrm{p}<$ 0.05 .

\section{Results}

A total of 110 individuals were enrolled in our study. The average age of patients with PCOS $29.5 \pm 5.1$ was years, with the height $162 \pm 6$ and weight $62.3 \pm 9.7$, control subjects were $26 \pm 5.2$ years $1.61 \pm 6.18$ height and $73.1 \pm 19.5$ weight was for control subjects. Comparison of the study groups concerning study variables is summarized in Table 1. The body mass index $\left(\mathrm{kg} / \mathrm{m}^{2}\right)$ was $38.4 \pm 5.8$ for the control group and $30.1 \pm 12.8$ with a mean (SD) for the PCOS group. To evaluate and quantify hirsutism in women the Ferriman-Gallwey (FG) score was calculated, it was $6.7 \pm 5.1$ for the control group and $13.9 \pm 7$ for the PCOS group. Furthermore, 49 of ( $84.48 \%) 58$ women had ovulatory dysfunction with irregular ovulation condition but not completely absent (oligo) as well 9 of $(14.51 \%)$ PCOS diagnosed women were not having irregular ovulation condition. Additionally, $51(87.93 \%)$ of PCOS women had menstrual irregularity and 7 of $(12.06 \%)$ PCOS women were not having menstrual irregularity. Likewise, infertility was absent in 32 (54.23\%) PCOS women, but 11 had (18.64\%) infertility, 10 women $(16.94 \%)$ state were unknown, 4 women were $(6.77 \%)$ unmarried and 1 was $(1.69 \%)$ virgin. Withal, 34 $(58.62 \%)$ of PCOS women had PCOS in the family but not have $24(41.37 \%)$ of them PCOS in the family. 38 $(65.51 \%)$ of our participants were treated with drugs and 20 of them $(34.48 \%)$ were not used any drug. Also, 30 $(51.72 \%)$ of the PCOS group had a history of cardiac disease in their family but $28(48.27 \%)$ of them had not. Finally, $13(22.43 \%)$ of PCOS women had additional diseases and $45(77.58 \%)$ of them had not any additional disease. 
Table 1. Baseline characteristics of study participants for PCOS $n=65$ and $n=45$ for the control group.

\begin{tabular}{|c|c|c|}
\hline \multicolumn{3}{|c|}{ Baseline characteristics of study participants } \\
\hline & Control & PCOS \\
\hline $\mathbf{N}$ & 45 & 65 \\
\hline Age & $29.5 \pm 5.12$ & $26 \pm 5.25$ \\
\hline Height(cm) & $162 \pm 6.03$ & $1.61 \pm 6.18$ \\
\hline Weight (kg) & $62.3 \pm 9.78$ & $73.1 \pm 19.5$ \\
\hline Body Mass Index (kg/m2) & $30.1 \pm 12.8$ & $38.4 \pm 5.87$ \\
\hline \multicolumn{3}{|l|}{ Oligo/Anovulation } \\
\hline Present & Null & $84.48 \%$ \\
\hline Absent & Null & $14.51 \%$ \\
\hline \multicolumn{3}{|l|}{ Menstrual irregularity } \\
\hline Present & Null & $87.93 \%$ \\
\hline Absent & Null & $12.06 \%$ \\
\hline \multicolumn{3}{|l|}{ Infertility } \\
\hline Absent & $35(83.33 \%)$ & $54.23 \%$ \\
\hline Present & Null & $18.64 \%$ \\
\hline Unknown & $7(16.66 \%)$ & $16.94 \%$ \\
\hline Unmarried & Null & $6.77 \%$ \\
\hline Virgin & Null & $1.69 \%$ \\
\hline Ferriman-Gallwey (FG) score & $6.7 \pm 5.1$ & $13.9 \pm 7$ \\
\hline \multicolumn{3}{|l|}{ Hyperandrogenemia } \\
\hline Present & Null & $81,03 \%$ \\
\hline Absent & Null & $18,96 \%$ \\
\hline \multicolumn{3}{|l|}{ PCOS in the family } \\
\hline Absent & Null & $41.37 \%$ \\
\hline Present & Null & $58.62 \%$ \\
\hline \multicolumn{3}{|l|}{ Treatment (Drugs) } \\
\hline Absent & Null & $65.51 \%$ \\
\hline Present & Null & $34.48 \%$ \\
\hline \multicolumn{3}{|c|}{ History of cardiac disease in the family } \\
\hline Absent & Null & $48.27 \%$ \\
\hline Present & Null & $51.72 \%$ \\
\hline \multicolumn{3}{|l|}{ Additional disease } \\
\hline Absent & Null & $77.58 \%$ \\
\hline Present & Null & $22.43 \%$ \\
\hline
\end{tabular}

In Fig.1 we compared the hormone concentration of PCOS women and control subjects. Women with PCOS had high levels of Luteinizing hormone (LH) (IU/L) secretions (10.5 \pm 6.3 ) which was significantly $(p<0.01)$ higher than the control group $(5.3 \pm 2.5)$. High levels of LH contribute to the high levels of androgens (male hormones such as testosterone), and this along with low levels of follicle-stimulating hormone (FSH) (IU/L) secretion (5.5 \pm 1.7$)$ compared to the control group (6.8 \pm 3.6$)$ that contributes to poor egg development and an inability to ovulate. The lack of ovulation alters levels of estrogen, FSH, and LH. Estradiol $(\mathrm{nmol} / \mathrm{L})$ level $(44.9 \pm 46)$ was lower than the control group $(72.6 \pm 10)$ with the significant $\mathrm{p}$-value $(\mathrm{p}<0.001)$, while androgen levels Dehydroepiandrosterone sulfate (DHEAS) (nmol/L) (276 \pm 89.8$)$ was higher than usual compared to the control group (191 \pm 50.5$)$. Prolactin (PRL) level was elevated in PCOS subjects $(16.8 \pm 8.56)$ too compared to the control group $(14.1 \pm 10.6)$ as seen in Table 2. Extra male hormones disrupt the menstrual cycle, so women with PCOS get fewer periods than usual. Most women with PCOS are surprised to find that their estrogen levels fall within the normal range (about $25-75 \mathrm{pg} / \mathrm{ml}$ ). This may be because the high levels of insulin and testosterone found in women with PCOS are sometimes converted to estrogen. High levels of estrogen are known as estrogen dominance and can occur in women with PCOS disease. PCOS is a hormone imbalance that can cause irregular periods, unwanted hair growth, and acne. Also, we measured Thyroidstimulating hormone (TSH) levels both in the control and PCOS group, there was an elevation of TSH level in the PCOS group (2.4 \pm 1.5$)$ compared to the control group (1.8 \pm 1.6$)$. 
Table 2. The mean and SD of hormonal parameters in PCOS and healthy control women $(n=65, n=45)$.

\begin{tabular}{|l|l|l|}
\hline Hormonal parameters & Control & PCOS \\
\hline Dehydroepiandrosterone sulfate (DHEAS) (nmol/L) & $191 \pm 50.5$ & $276 \pm 89.8$ \\
\hline Follicle-stimulating hormone (FSH) (IU/L & $6.86 \pm 3.6$ & $5.53 \pm 1.72$ \\
\hline Luteinizing hormone (LH) (IU/L) & $5.35 \pm 2.5$ & $10.5 \pm 6.38$ \\
\hline Estradiol (nmol/L) & $72.6 \pm 10$ & $44.9 \pm 46$ \\
\hline Thyroid-stimulating hormone (TSH) & $1.85 \pm 1.63$ & $2.44 \pm 1.54$ \\
\hline PRL & $14.1 \pm 10.6$ & $16.8 \pm 8.56$ \\
\hline LH/FSH & $2.1 \pm 2.19$ & $0.90 \pm 0.45$ \\
\hline
\end{tabular}

Finally, related to PCOS disease in young women, our results show that the lack of ovulation alters levels of estrogen, progesterone, FSH, and LH. Estrogen and progesterone levels are lower than usual, while androgen levels are higher than usual as in previous studies. Estrogens are a group of steroid hormones that are lipids made up of four fused rings of carbon. Also, Progesterone (P4) and Testosterone (T) are steroid form hormones. Follicle-stimulating hormone (FSH) and Luteinizing hormone (LH) is a glycoprotein structured hormone. Prolactin (PRL) Human prolactin is a non-glycosylated protein, which contains a simple polypeptide chain of 198 amino acids. Comparatively, LH/FSH levels were higher than the Control group but lower than 2.5 in PCOS women.

As shown in Table 3, Oral Glucose Tolerance Test (OGTT) (128.3 \pm 33 ) was lower than $140 \mathrm{mg} / \mathrm{dl}$ on the other hand two women (3.44\%) had Type II diabetes. HOMA IR was elevated in PCOS women (8.2 \pm 15$)$ compared to the control group (2.1 \pm 0.8$)$.

\begin{tabular}{|l|l|l|}
\hline Metabolic parameters & Control & PCOS \\
\hline Oral Glucose Tolerance Test (OGTT) & $120.38 \pm 13.2$ & $128.33 \pm 33.8$ \\
\hline Diabetes metabolic parameters & Null & Type II, $2(3.44 \%)$ \\
\hline HOMA IR & $2.12 \pm 0.88$ & $8.26 \pm 15.06$ \\
\hline
\end{tabular}

Table 3. Shows mean and SD of metabolic parameters in patients with, PCOS and controls.

Additionally, $65.51 \%$ of the patients with PCOS were not used specific drug treatments. Totally 18 (31.03\%) PCOS patients were used specific drugs for the treatment of metabolic disease. As indicated in Table 4, the participants were using different drugs such as yasmin, oxytocin, and additionally 13 patients had different diseases.

\begin{tabular}{lclc}
\hline \multicolumn{1}{l}{ Drugs for the treatment of PCOS } & \multicolumn{1}{c}{ Additional Diseases } \\
Yasmin & $5(8.62 \%)$ & Hypothyroidy & $3(5.17 \%)$ \\
Oxytocin & $3(5.17 \%)$ & Guatr & $2(3.44 \%)$ \\
Diane & $2(3.44 \%)$ & Hypercholesterolemia & $2(3.44 \%)$ \\
Qlairista & $2(3.44 \%)$ & Asthma & $2(3.44 \%)$ \\
Lazer & $2(3.44 \%)$ & Type2 Diabetes & $2(3.44 \%)$ \\
Andropor & $1(1.72 \%)$ & Psoriasis & $1(1.72 \%)$ \\
Glifor & $1(1.72 \%)$ & Hydronephrosis & $1(1.72 \%)$ \\
Duphastone & $1(1.72 \%)$ & & \\
Inofolic & $1(1.72 \%)$ & & \\
\hline
\end{tabular}

Table 4. Drug treatments and additional diseases of PCOS patients. $\mathrm{n}=58$.

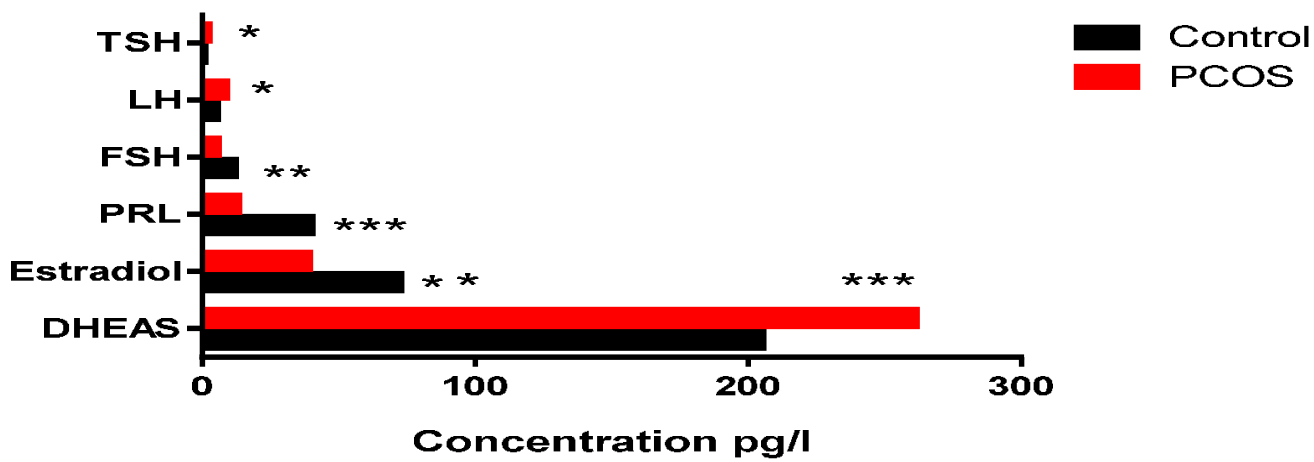

Figure 1. Hormone concentrations $(\mathrm{pg} / \mathrm{l})$. Black color represents control group $(\mathrm{n}=45)$; red color represents PCOS group $(n=65)$. Tested with analysis of variance ANOVA followed with post-hoc analysis. A significant difference was expressed as a p-value which was calculated by one-way ANOVA test followed by Bonferroni's post hoc test. $\mathrm{p}$ was considered significant at $<0.05$. Significance levels: $* \mathrm{p}<0.05, * * * \mathrm{p}<0.001$ vs. control group. 
As illustrated in PCOS patient's TNF-alpha levels were higher $(226.3 \pm 43)$ than control $(80.02 \pm 26.48)$ with a significant $\mathrm{p}$-value $(\mathrm{p}<0.001)$. IL-6 level in PCOS women compared to the controls too. There was an elevation in PCOS women $(346.9 \pm 15.71)$ compared to the control group $(180.6 \pm 55.51)$. Together, these alterations cytokine and oxidative stress status imbalance contribute to inhibition on TNF- $\alpha$ or IL-6 acting to modify the metabolism under PCOS condition (Figure 2).
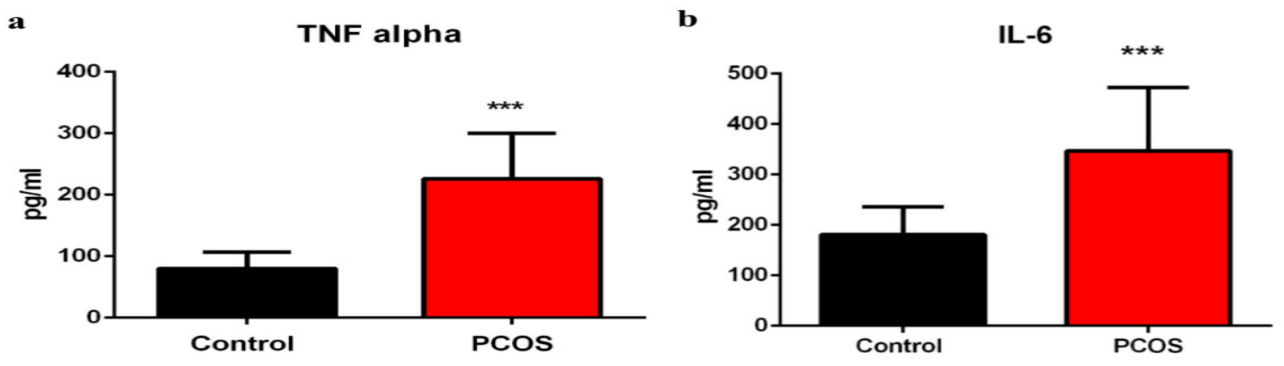

Figure 2. Cytokine level TNF-alpha (pg/ml) and IL-6 (pg/ml) and oxidative stress status measured in the blood serums. Groups are presented in legends. Data are presenting mean $( \pm \mathrm{SD})$ values. The total number of subjects in each group: $\mathrm{n}$ control $=45, \mathrm{n}$ PCOS $=65$. Significance levels: $* * * \mathrm{p}<0.01$ control vs. PCOS group.

The only statistically significant results from the Pearson correlation test obtained from Cytokine levels and laboratory test conditions were presented in Figure 2. As is shown, a positive and negative correlation was obtained. A positive correlation means, that if we observed an increase of the first factor, the second one also increases, while negative correlations mean, that if we observed an increase of the first factor, the second one decreases. When we compared results obtained for control and PCOS samples, differences in the kind and values of correlation, was visible. In the control samples negative correlation between IL-6 and DHEAS levels in control patients was obtained, while in PCOS samples, this correlation was not noticed. However, in control samples, there was no correlation between TNF alpha and IL-6, while in the PCOS samples positive correlation, was obtained.

\section{Discussion}

In this study, we highlighted the significant role of IL-6 and TNF- alpha as important inflammatory cytokines and androgenic hormone DHEAS, as markers with PCOS disease. As it is known LH/FSH $>2.5$ is considered as PCOS disease, but our results showed that women with PCOS may have LH/FSH level $<2.5$. but this ratio was higher than control women as in previous studies (16). Our correlation analysis signified important points. As a pro-inflammatory marker, we measured IL-6 level and inflammatory marker TNF-alpha both in PCOS and healthy women, the results showed that IL-6 and TNF-alpha correlate in PCOS women but not in healthy women as previous studies reported both parameters may have a role in PCOS related disease (17). IL-6 level has a significant difference between PCOS and control women. Also, when we take a cut-off between 20-25 and 23-30 age women, IL-6 had a rise with advancing age both in PCOS and healthy women. Previous studies had shown that IL- 6 may rise with advancing age (18), our results showed that IL-6 tends to be a rise in PCOS women too. It is known that IL-6 is a cytokine that is being produced either by immune cells or nonimmune cells some typically endocrine which have pathophysiologic roles in humans. Ref. Recent studies have shown that IL-6 and TNF-alpha are the product of adipose tissue claimed that they may have an important role in lipid metabolism $(19,20)$. IL-6 is a cytokine that is regulated by hormones. The metabolic product of IL-6 and hormones has a correlation and IL-6 has many endocrine effects for instance it has a role in the stimulation of the hypothalamicpituitary-adrenal axis during inflammatory stress and has influence intermediary metabolism $(\underline{21}, \underline{22})$. Ersler et al, showed that IL- 6 with the age-associated increase is largely prevented by life span-prolonging dietary control (18). This result suggests dietary control may have related to IL-6 level and maybe a therapeutic approach for PCOS women. Contrary to previous findings our clinical reports and experiments obtained from PCOS women support and extend the major role that IL-6 plays in metabolism and endocrine. As an additional contribution, we find that IL-6 may correlate with DHEAS hyperandrogenic hormone as previous studies (23). Furthermore, LH and DHEAS hormone levels were significantly correlated with IL-6 levels. Interestingly DHEAS and IL- 6 have a correlation in healthy women but not in PCOS women (24). Additionally, ANOVA and following Bonferroni multiple comparisons showed that DHEAS level had significant changes between groups and among subjects in PCOS women and control women. DHEAS level tended to be higher in PCOS women. Also, there was a correlation between age, BMI, IL-6 level, and DHEAS level as recent studies (25). As a result, our results suggest that endocrine hormone concentrations were significantly higher in PCOS. Finally, PCOS is generally considered as a metabolic disorder, researchers may focus on IL-6 as an inflammatory marker and DHEAS as a 
hyperandrogenic hormone and maybe the relation between these markers since that may have an important role in the management of the disease and thus may improve women's health. the hormone may have an impact on the metabolic disorder.

\section{Conclusion}

In conclusion, PCOS is a gender-specific complicated endocrine disorder relating to defects in psychological, metabolic, and reproductive functions. Clinical reports are important traces for the consideration of the disease. IL-6 and DHEAS levels showed alterations among and between PCOS and healthy participants depending on age and BMI. There is a low-grade inflammation in PCOS women.

Therefore, as a result, IL-6 and TNF- alpha rises against low-grade inflammation in the PCOS can be found and there is a correlation with DHEAS with IL-6 level. Furthermore, well-planned prospective research is required to explore more about androgens and cytokines.

\section{Authors' Contributions}

All authors made substantial contributions to conception and design, and/or acquisition of data, and/or analysis and interpretation of data; participated in drafting the article or revising it critically for important intellectual content, and gave final approval of the version to be submitted.

\section{Author Disclosure Statement}

The authors declare no conflicts of interest that could be perceived as prejudicing the impartiality of the research reported.

\section{Funding Information}

No funding was received for this article

\section{References}

1. Adibhatla RM, Dempsy R, Hatcher JF. (2008). Integration of cytokine biology and lipid metabolism in stroke, Front. Biosci. 13:1250-1270 https://doi.org/10.2741/2759.

2. Azziz R, Carmina E, Chen Z, Dunaif A, Laven JS, Legro RS, Lizneva D, Natterson-Horowtiz B, Teede HJ, Yildiz BO. (2016). Polycystic ovary syndrome. Nat Rev Dis Primers. 2:16057 https://doi.org/10.1038/nrdp.2016.57.

3. Barton BE. (1997). IL-6: Insights into novel biological activities, Clin. Immunol. Immunopathol. 85:16-20 https://doi.org/10.1006/clin.1997.4420.

4. Cardoso NS, Ribeiro VB, Dutra SGV, Ferriani RA, Gastaldi AC, Araújo JE, Souza HCD. (2020). Polycystic ovary syndrome associated with increased adiposity interferes with serum levels of TNF-alpha and IL-6 differently from leptin and adiponectin. Arch Endocrinol Metab. 64 (1): 4-10 DOI: 10.20945/23593997000000197

5. Cavaillon JM, Haeffner-Cavaillon N. (1993). Cytokines et inflammation., Rev. Du Prat, 43:547-552 PMID: 11502077

6. Didion SP. (2017). Cellular and oxidative mechanisms associated with interleukin-6 signaling in the vasculature, Int. J. Mol. Sci.18 https://doi.org/10.3390/ijms18122563.

7. Emidio GD, Placidi M, Rea F, Rossi G, Falone S, Cristiano L, Nottola S, D'Alessandro AM, Amicarelli F, Palmerini MG, Tatone C. (2020). Methylglyoxal-Dependent Glycative Stress and Deregulation of SIRT1 Functional Network in the Ovary of PCOS Mice. Cells, 9(1): E209 https://doi.org/10.3390/cells9010209.

8. Ershler WB, Sun WH, Binkley N, Gravenstein S, Volk MJ, Kamoske G, Klopp RG, Roecker EB, Daynes RA, Weindruch R. (1993). Interleukin-6 and aging: blood levels and mononuclear cell production increase with advancing age and in vitro production is modifiable by dietary restriction. Lymphokine Cytokine Res. 12(4):225-30

9. Ganie MA, Sahar T, Rashid A, Wani IA, Nisar S, Sathyapalan T, Vishnubhatla S, Ramakrishnan L, Parvez T, Geer I. (2019). Comparative Evaluation of Biomarkers of Inflammation Among Indian Women with Polycystic Ovary Syndrome (PCOS) Consuming Vegetarian vs. Non-vegetarian Diet. Front Endocrinol (Lausanne).10:699. https://doi.org/10.3389/fendo.2019.00699.

10. Ghanemi A, St-Amand J. (2018). Interleukin-6 as a "metabolic hormone," Cytokine. 112:132-136 https://doi.org/10.1016/j.cyto.2018.06.034.

11. Hotamisligil GS, Arner P, Caro JF, Atkinson RL, Spiegelman BM. (1995). Increased adipose tissue expression of tumor necrosis factor- in human obesity and insulin resistance. J Clin Invest. 95:2409-2415

12. Huang J, Upadhyay UM, Tamargo RJ. (2006). Inflammation in stroke and focal cerebral ischemia, Surg. Neurol. 66:232-245 https://doi.org/10.1016/j.surneu.2005.12.028.

13. Judd AM, Call GB, Barney M, McIlmoil CJ, Balls AG, Adams A, Oliveira GK. (2000). Possible function of 
IL-6 and TNF as intraadrenal factors in the regulation of adrenal steroid secretion. Ann N Y Acad Sci. 917:628-37 doi: 10.1111/j.1749-6632.2000.tb05428.x

14. March WA, Moore VM, Willson KJ, Phillips DI, Norman RJ, Davies MJ. (2010). The prevalence of polycystic ovary syndrome in a community sample assessed under contrasting diagnostic criteria. Hum Reprod. 25; (2): 544-51https://doi.org/10.1093/humrep/dep399.

15. Mastorakos G, Chrousos GP, Weber JS. (1993).Recombinant interleukin-6 activates the hypothalamicpituitary-adrenal axis in humans. J Clin Endocrinol Metab. 77:1690-1694

16. Mohammadi M. (2019). Oxidative stress and polycystic ovary syndrome: a brief review. Int J Prev Med. 10:86 https://doi.org/10.4103/ijpvm.\%20IJPVM_576_17.

17. Murri M, Luque-Ramírez M, Insenser M, Ojeda-Ojeda M, Escobar-Morreale HF. (2013). Circulating markers of oxidative stress and polycystic ovary syndrome (PCOS): a systematic review and meta-analysis. Hum Reprod. Update. 19(3):268-88https://doi.org/10.1093/humupd/dms059.

18. S.M. Opal, V.A. DePalo. (2000). Anti-inflammatory cytokines, Chest. $117 \quad 1162-1172$ https://doi.org/10.1378/chest.117.4.1162.

19. Saadia Z. (2020). Follicle Stimulating Hormone (LH: FSH) Ratio in Polycystic Ovary Syndrome (PCOS) Obese vs. Non- Obese Women. Med Arch.74(4):289-293 doi: 10.5455/medarh.2020.74.289-293

20. Saghizadeh M, Ong JM, Garvey WT, Henry RR, Kern PA. (1996). The expression of TNF-alpha by human muscle. Relationship to insulin resistance. J Clin Invest. 97: 1111-1116

21. Straub RH, Hense HW, Andus T, Scholmerich J, Riegger GAJ, Shunkert H. (2000). Hormone replacement therapy and interrelation between serum interleukin-6 and body mass index in postmenopausal women: a population study. J Clin Endocrinol Metab. 85:1340-1344

22. Tapia G, Fernández V, Varela P, Cornejo P, Guerrero J, Videla LA. (2003). Thyroid hormone-induced oxidative stress triggers nuclear factor- $\mathrm{\kappa B}$ activation and cytokine gene expression in rat liver, Free Radic. Biol. Med. 35:257-265 https://doi.org/10.1016/S0891-5849(03)00209-0.

23. Viviani B. (2004). Cytokines role in neurodegenerative events. Toxicol. Lett.; 149:85-89 https://doi.org/10.1016/j.toxlet.2003.12.022.

24. Wei X, Zhang J, Peng W, Xu H, Wei Z, Pang L, Liu J, Wang T. (2020). Interleukin-6 increases adrenal androgen release by regulating the expression of steroidogenic proteins in NCI-H295R cells. J Cell Physiol. ;235(12):9432-9444 DOI: 10.1002/jcp.29748

25. Wu G, Hu X, Ding J, Yang J. (2020). The effect of glutamine on Dehydroepiandrosterone-induced polycystic ovary syndrome rats. J Ovarian Res.13(1):57 DOI: 10.1186/s13048-020-00650-7 